\title{
The joint Rouse-Zimm theory of the dynamics of polymers in dilute solutions
}

\author{
V.Lisy ${ }^{1}$, J.Tothova ${ }^{1}$, A.Zatovsky ${ }^{2}$ \\ 1 Institute of Physics, P.J.Safarik University, \\ Jesenna 5, 04154 Kosice, Slovakia \\ 2 Department of Theoretical Physics, \\ I.I.Mechnikov Odessa National University, \\ 2, Dvoryanskaya Str., 65026 Odessa, Ukraine
}

Received July 11, 2005

\begin{abstract}
We propose a theory of the dynamics of polymers in dilute solution, in which the popular Zimm and Rouse models are just the limiting cases of an infinitely large and small draining parameter. The equation of motion for the polymer segments (beads) is solved together with Brinkman's equation for the solvent velocity that takes into account the presence of other polymer coils in the solution. The equation for the polymer normal modes is obtained and the relevant time correlation functions are found. A tendency to the time-dependent hydrodynamic screening is demonstrated on the diffusion of the polymers as well as on the relaxation of their internal modes. With the growing concentration of the coils in the solution, they both show a transition to the exactly Rouse behaviour. The shear viscosity of the solution, the Huggins coefficient and other quantities are calculated and shown to be notably different from the known results.
\end{abstract}

Key words: polymers, dynamics, dilute solution, rheology

PACS: $36.20 . F z, 36.20 .-r, 82.35 . L r, 82.37 .-j, 83.80 . R s$

\section{Introduction}

Interest in dilute polymer solutions arises primarily from their importance in the characterization of polymers, their interaction with solvent and from a fundamental interest in understanding macromolecular response to hydrodynamic forces, free from the complications of intermolecular entanglements. Despite five decades of investigations, these issues are not entirely understood. So, there is a systematic discrepancy between the dynamic scattering data and the theory [1-3]. The existing theories give different results for the viscosity of dilute polymer solutions (see, e.g., $[4,5]$ and the citations therein), the observed monomer motion in single polymer chains cannot be explained by the available theories [6], the time dependence of hydrodynamic screening in solutions has not been explained [7], etc. For other problems we refer the reader to the recent review [8]. The aim of this work was to contribute to the solution of some of these problems by developing a phenomenological bead-spring theory of diffusion of an individual "test" polymer and the relaxation of its internal modes in the solution of unentangled polymers. Our approach differs from the traditional ones in several main points. Firstly, the joint Rouse-Zimm theory is exploited [9]. In the literature, following de Gennes [10], it is assumed that at $\theta$ conditions, the Zimm modes with the dispersion of relaxation times $\tau_{p Z} \sim p^{-3 / 2}$, where $p$ is the mode number, at low frequencies, should always dominate the Rouse modes with $\tau_{p \mathrm{R}} \sim p^{-2}$; we show that these both contributions to the polymer characteristics should be taken into account. Next, the internal modes are distributed discretely (the assumption of their continuous distribution with respect to $p$ is true only in a restricted time domain and often leads to incorrect interpretation of experimental data [11]. The formalism from our theory with the time-dependent hydrodynamics of polymers has been adopted $[9,12]$ and, finally, the Brinkman's theory $[13,14]$ for the flow in porous media is used in order to take into account the effect of other coils on the test polymer. The presented theory has the following limitations. The considered time scales are $t \gg \tau_{\mathrm{R}}=R^{2} \rho / \eta$, where $R$ is the hydrodynamic radius 
of the polymer, $\rho$ is the density and $\eta$ is the viscosity of the solvent. This means that the effects of hydrodynamic memory (or the viscous aftereffect) are neglected $[9,12,15]$. The distribution of the coils in solution is considered to be stationary (this is justified at least for the times $t \ll \tau_{D}$; the choice of this time scale is possible since always $\tau_{p} \ll \tau_{D}$, where $\tau_{D}=R^{2} / D$ is the characteristic time of the coil diffusion with the diffusion coefficient $D$ ). Our theory is also restricted to $\theta$ solvents [4]; generalizations to other cases require the knowledge of the equilibrium distribution of the segments when the exclude volume interactions are taken into account. As already mentioned, only solutions of unentangled polymers are considered. We are thus limited to the concentrations of the chains $c<1 /[\eta]$, where $[\eta]$ is the intrinsic viscosity and $c$ is the number of polymers per unit volume [8]. In spite of all these restrictions and other ones, like those that we do not consider, i. e., the internal viscosity of polymers and the self-entanglements (the importance and even the reality of these interactions are uncertain [8]), we believe that the results of our theory could be of interest. In particular, we have found new expressions for the quantities describing the behaviour of flexible polymers in solution, such as the diffusion coefficient of the coil, the relaxation times of the internal modes, the viscosity of the solution, and the Huggins coefficient. These quantities have been obtained from a generalized Rouse-Zimm equation for the position vectors of the polymer segments and the Oseen tensor describing the velocity field of the solvent due to perturbation. Finally, our theory describes in a simple manner the hydrodynamic screening, i.e. the concentration and time-dependent transition between the Zimm and (as distinct from the previous theories) the exact Rouse behaviour of the polymer.

\section{The Rouse-Zimm-Brinkman theory of polymer dynamics}

We choose one coil as a "test" polymer. The equation of motion of its $n$th segment is

$$
M \frac{\mathrm{d}^{2} \vec{x}_{n}(t)}{\mathrm{d} t^{2}}=\vec{f}_{n}^{\mathrm{fr}}+\vec{f}_{n}^{\mathrm{ch}}+\vec{f}_{n}
$$

Here, $\vec{x}_{n}$ is the position vector of the segment (a spherical bead) from the $N$ ones constituting the polymer, $M$ is the bead mass, $\vec{f}_{n}^{\text {ch }}$ is the force from the neighboring beads along the chain, $\vec{f}_{n}$ is the random force due to the motion of the molecules of solvent, and $\vec{f} \underset{n}{\mathrm{fr}}$ is the Stokes friction force on the bead during its motion in the solvent $[4,16]$ :

$$
\vec{f}_{n}^{\mathrm{fr}}=-\xi\left[\frac{\mathrm{d} \vec{x}_{n}}{\mathrm{~d} t}-\vec{v}\left(\vec{x}_{n}\right)\right],
$$

where $\vec{v}$ denotes the velocity of the solvent in the place of the $n$th bead due to the motion of other beads. The friction coefficient on the bead with radius $b$ is $\xi=6 \pi \eta b$. This expression holds in the case of steady flow and takes into account the hydrodynamic interaction. In a more general case, with the hydrodynamic memory $[9,12,15]$ the force $(2)$ should be replaced by the Boussinesq force and equation (1) has to be solved together with the nonstationary hydrodynamic equations for the macroscopic velocity of the solvent. To take into account the presence of other polymers in solution, we use the Brinkman's work [13] (see also [14]) in which a polymer is considered as a porous medium. In our approach, the whole solution is such a medium while the coils are obstacles to the solvent flow. Then, in the right hand part of the Navier-Stokes equation, a term $-\kappa^{2} \eta \vec{v}$ has to be added, where $\kappa^{-2}$ is the solvent permeability. This term has the sense of an average value of the force acting on the liquid in the element of volume $d V$, provided the average number of polymers in solution per unit volume is $c$; then $\kappa^{2} \eta=c f$, where $f$ is the friction factor on one coil. Thus, for an incompressible solvent $(\nabla \vec{v}=0)$ we have to solve the equation

$$
\rho \frac{\partial \vec{v}}{\partial t}=-\nabla p+\eta \triangle \vec{v}-\kappa^{2} \eta \vec{v}+\vec{\varphi}
$$

Here $p$ is the pressure and $\vec{\varphi}$ is the density of the force from the beads of the studied polymer acting on the solvent [16],

$$
\vec{\varphi}(\vec{x})=-\sum_{n} \vec{f}_{n}^{\mathrm{fr}}\left(\vec{x}_{n}\right) \delta\left(\vec{x}-\vec{x}_{n}\right)
$$


To solve this equation is a difficult problem since the polymer chains are mobile. However, restricting ourselves to the times much shorter than $\tau_{D}$, the concentration $c$ can be assumed constant. The above equations then describe the motion of one bead in the solvent with the effective action of other coils on the motion of the solvent flow. This problem can be transformed to that solved already in [17] (see also [9,15]). The velocity field can be in the Fourier representation in the time written as follows:

$$
v_{\alpha}^{\omega}(\vec{r})=\int \mathrm{d} \vec{r}^{\prime} \sum_{\beta} H_{\alpha \beta}^{\omega}\left(\vec{r}-\vec{r}^{\prime}\right) \varphi_{\beta}^{\omega}\left(\vec{r}^{\prime}\right) .
$$

Here the analog of the Oseen tensor is

$$
\begin{gathered}
H_{\alpha \beta}^{\omega}(\vec{r})=A \delta_{\alpha \beta}+B r_{\alpha} r_{\beta} r^{-2}, \\
A=(8 \pi \eta r)^{-1}\left\{\mathrm{e}^{-y}-y\left[\left(1-\mathrm{e}^{-y}\right) y^{-1}\right]^{\prime \prime}\right\}, \\
B=(8 \pi \eta r)^{-1}\left\{\mathrm{e}^{-y}+3 y\left[\left(1-\mathrm{e}^{-y}\right) y^{-1}\right]^{\prime \prime}\right\},
\end{gathered}
$$

$y=r \chi, \chi^{2}=\kappa^{2}-\mathrm{i} \omega \varrho / \eta$, and the prime means the differentiation with respect to $y$. In the particular case $\omega=0$ and for permeable solvent, $\kappa=0$, equation (6) coincides with the well-known result of Zimm [4]. Using this solution, a generalization of the Rouse-Zimm equation can be obtained from the equation of motion [9]. The preaveraging of the Oseen tensor over the equilibrium Gaussian distribution of the beads $[4,16]$ gives

$$
\begin{aligned}
\left\langle H_{\alpha \beta n m}^{\omega}\right\rangle_{0} & =\delta_{\alpha \beta} h^{\omega}(n-m), \quad \vec{r}_{n m} \equiv \overrightarrow{x_{n}}-\overrightarrow{x_{m}}, \\
h^{\omega}(n-m) & =\left(6 \pi^{3}|n-m|\right)^{-1 / 2}(\eta a)^{-1}\left[1-\sqrt{\pi} z \exp \left(z^{2}\right) \operatorname{erfc}(z)\right] .
\end{aligned}
$$

Here $a$ is the mean square distance between the beads along the chain and $z \equiv \chi a(|n-m| / 6)^{1 / 2}$. Then in the continuum approximation with respect to the variable $n$ the new Rouse-Zimm equation reads

$$
\begin{aligned}
-\mathrm{i} \omega \vec{x}^{\omega}(n)= & \frac{1}{\xi}\left[\frac{3 k_{\mathrm{B}} T}{a^{2}} \frac{\partial^{2} \vec{x}^{\omega}(n)}{\partial n^{2}}+M \omega^{2} \vec{x}^{\omega}(n)+\vec{f}^{\omega}(n)\right] \\
& +\int_{0}^{N} \mathrm{~d} m h^{\omega}(n-m)\left[\frac{3 k_{\mathrm{B}} T}{a^{2}} \frac{\partial^{2} \vec{x}^{\omega}(m)}{\partial m^{2}}+M \omega^{2} \vec{x}^{\omega}(m)+\vec{f}^{\omega}(m)\right] .
\end{aligned}
$$

It is solved with the help of the Fourier transformation (FT) in $n$, taking into account the boundary conditions at the ends of the chain [16], $\partial \vec{x} / \partial n=0$ at $n=0, N: \vec{x} \omega(n)=\vec{y}_{0}^{\omega}+$ $2 \sum_{p \geqslant 1} \vec{y}_{p}^{\omega} \cos (\pi n p / N)$. The inverse FT then yields

$$
\vec{y}_{p}^{\omega}=\vec{f}_{p}^{\omega}\left[-\mathrm{i} \omega \Xi_{p}^{\omega}-M \omega^{2}+K_{p}\right]^{-1}
$$

where

$$
\Xi_{p}^{\omega}=\xi\left[1+\left(2-\delta_{p 0}\right) N \xi h_{p p}^{\omega}\right]^{-1}, \quad K_{p}=3 k_{\mathrm{B}} T\left(\frac{\pi p}{N a}\right)^{2}, \quad p=0,1,2, \ldots
$$

and the Oseen matrix is $[9,15]$

$$
\begin{aligned}
h_{p p}^{\omega} & =\frac{1}{\pi \eta a \sqrt{3 \pi N p}} \frac{1+\chi_{p}}{1+\left(1+\chi_{p}\right)^{2}}, \quad \chi_{p} \equiv \sqrt{\frac{N}{3 \pi p}} \chi a, \quad p=1,2, \ldots, \\
h_{00}^{\omega} & =\frac{2}{\sqrt{6 N} \pi \eta a} \frac{1}{\chi_{\omega}}\left[1-\frac{2}{\sqrt{\pi} \chi_{\omega}}+\frac{1}{\chi_{\omega}^{2}}\left(1-\exp \chi_{\omega}^{2} \operatorname{erfc} \chi_{\omega}\right)\right], \quad \chi_{\omega} \equiv \sqrt{\frac{N}{6}} \chi a .
\end{aligned}
$$

Using the fluctuation-dissipation theorem, the time correlation functions of the normal modes are

$$
\psi_{p}(t)=\left\langle y_{\alpha p}(0) y_{\alpha p}(t)\right\rangle=\frac{k_{\mathrm{B}} T}{\left(2-\delta_{p 0}\right) \pi N} \int_{-\infty}^{\infty} \mathrm{d} \omega \cos \omega t \frac{\operatorname{Re} \Xi_{p}^{\omega}}{\left|-\mathrm{i} \omega \Xi_{p}^{\omega}-M \omega^{2}+K_{p}\right|^{2}}
$$




\subsection{Diffusion of the coil}

In the stationary limit $\omega=0$ so that $\chi=\kappa$. Then the preaveraged Oseen tensor (6) is

$$
\left\langle H_{\alpha \beta}^{\omega}\right\rangle_{0}=\left\langle\frac{\exp (-\chi r)}{r}\right\rangle_{0}
$$

Thus, the quantity $1 / \kappa$ (for small $\kappa r$ only) can be considered as a screening length. For an individual polymer we have $(p=0$ in equation (14)) [9]

$$
\psi_{0}(0)-\psi_{0}(t)=D t
$$

with the diffusion coefficient $D=D_{\mathrm{R}}+D_{\mathrm{Z}}(R$ and $Z$ denote the well-known Rouse and Zimm limits [4]. Now instead of equation (12) we have $h_{00}^{0}$ with $\chi_{0}=\kappa R_{\mathrm{G}}\left(R_{\mathrm{G}}\right.$ being the gyration radius), the diffusion coefficient depends on the concentration of the coils $c$,

$$
D=D_{\mathrm{R}}+D_{\mathrm{Z}}(c)
$$

$\left(D_{\mathrm{Z}}(0)=D_{\mathrm{Z}}\right)$ and consists of the Rouse (independent of the presence of other polymers) and the Zimm contributions. The latter one can be expressed in the form

$$
D_{\mathrm{Z}}(c)=D_{\mathrm{Z}} f(c)
$$

where $f(c)$ is a "universal" function for every polymer:

$$
f(c)=\frac{3 \sqrt{\pi}}{4 \chi_{0}}\left[1-\frac{2}{\sqrt{\pi} \chi_{0}}+\frac{1}{\chi_{0}^{2}}\left(1-\exp \chi_{0}^{2} \operatorname{erfc} \chi_{0}\right)\right] .
$$

The dependence of the permeability on the concentration is estimated as follows. The friction coefficient in the quantity $\kappa^{2}=c f / \eta$ from equation (3) can be determined using the Einstein relation $D=k_{\mathrm{B}} T / f$. In such a picture

$$
\kappa^{2}=\frac{27 \sqrt{\pi}}{16} \frac{\widetilde{c}}{R_{\mathrm{G}}^{2}}\left(1+\frac{3}{4 \sqrt{2} h}\right)^{-1} .
$$

Then, the values of $\kappa$ and $\chi_{0}$ depend on the draining parameter $h=2(3 N / \pi)^{1 / 2} b / a$ (if $h \gg 1$, the dynamics is of the Zimm type, for $h \ll 1$ we deal with the Rouse polymers). The quantity $\widetilde{c} \equiv 4 \pi R_{\mathrm{G}}^{3} c / 3$ denotes the number of polymers per volume of a sphere with the radius $R_{\mathrm{G}}$. With the increase of $c$ the Zimm term decreases and for large $c$ (small permeability $\kappa$ when $\chi_{0} \gg 1$ ) it becomes $\sim 1 / \sqrt{c}$,

$$
D_{\mathrm{Z}}(c) \approx \frac{2 k_{\mathrm{B}} T}{\pi \eta N a^{2}} \frac{1}{\kappa}
$$

The realistic case of small $c$ corresponds to $\chi_{0}=\kappa R_{\mathrm{G}} \ll 1$ when

$$
D_{\mathrm{Z}}(c)=k_{\mathrm{B}} T h_{00}^{0}(c)=D_{\mathrm{Z}}\left(1-\frac{3}{8 \sqrt{\pi}} \kappa R_{G}+\cdots\right) \text {. }
$$

The concentration dependent correction to $D_{\mathrm{Z}}$ is thus proportional to $\sqrt{c}$ and differs from other results (compare [18] and citations there, where this correction is $\sim c$ ). The behaviour of a free polymer depends on the draining parameter $h$. If $h$ is large, the Zimm polymer (at $c=0$ ) with growing $c$ should change its behavior to the diffusion with the Rouse coefficient $D_{\mathrm{R}}$.

\subsection{Dynamics of internal modes}

In the stationary case $(\omega=0)$ and at zero concentration $(\kappa=0)$ the diagonal elements of the Oseen matrix are well known [4]. Now $h_{p p}^{0}$ from equation (13) depends on $c$. The internal modes 
relax exponentially as in previous theories, $\psi_{p}(t) \propto \exp \left(-|t| / \tau_{p}\right)$, but their relaxation rates consist of the Rouse contribution and the concentration-dependent Zimm part,

$$
\frac{1}{\tau_{p}(c)}=\frac{1}{\tau_{p \mathrm{R}}}+\frac{1}{\tau_{p \mathrm{Z}}(c)},
$$

where $\tau_{p \mathrm{R}}$ and $\tau_{p \mathrm{Z}}(0) \equiv \tau_{p \mathrm{Z}}$ are given in $[4,16]$ and

$$
\tau_{p Z}(c)=\frac{1}{2} \frac{1+\left(1+\chi_{p}\right)^{2}}{1+\chi_{p}} \tau_{p Z}
$$

which behaves as

$$
\tau_{p \mathrm{Z}}(c)=\tau_{p \mathrm{Z}}\left(1+\frac{N}{6 \pi p} \kappa^{2} a^{2}-\cdots\right)
$$

if $c \rightarrow 0$, and (although unrealistic), as $c \rightarrow \infty$ one has

$$
\tau_{p \mathrm{Z}}(c) \approx \frac{1}{2} \tau_{p \mathrm{Z}} \chi_{p}=\frac{\left(N a^{2}\right)^{2} \eta}{6 \pi k_{\mathrm{B}} T p^{2}} \kappa .
$$

Note that for the internal modes, the draining parameter depends on the mode number $p: h(p)=$ $\tau_{p \mathrm{R}} / \tau_{p \mathrm{Z}}=h / \sqrt{p}$. The "universal" dependence of $\tau_{p \mathrm{Z}}(c) / \tau_{p \mathrm{Z}}(0)$ on $\chi_{p}(24)$ indicates that with the growing $c$ every polymer shows a tendency to become the Rouse one.

\subsection{Steady state viscosity and the Huggins coefficient}

The shear viscosity of the solution can be calculated from the formula $[4,8,16]$

$$
\eta(c)=\eta+\frac{1}{2} k_{\mathrm{B}} T c \sum_{p=1}^{\infty} \tau_{p}(c) .
$$

Using equation (24), in the Rouse limit we have the familiar result [4] $\eta(c)-\eta=\pi N^{2} a^{2} b c \eta / 6$. In the Zimm limit at small concentrations

$$
\begin{aligned}
\frac{\eta(c)-\eta}{\eta} & =\frac{c}{2 \sqrt{3 \pi}}(\sqrt{N} a)^{3} \zeta\left(\frac{3}{2}\right)\left[1+c N a^{2} R_{\mathrm{Z}} \zeta^{-1}\left(\frac{3}{2}\right) \zeta\left(\frac{5}{2}\right)+\cdots\right] \\
& =0.425 c\left(N a^{2}\right)^{3 / 2}\left[1+0.140 c\left(N a^{2}\right)^{3 / 2}+\cdots\right]
\end{aligned}
$$

where $R_{Z}$ is the Zimm hydrodynamic radius [4] and $\zeta$ is the Riemann zeta function. The first term coincides with the known result [4]. A more general expression for the viscosity, following from equations (27) and (24), is

$$
\frac{\eta(c)-\eta}{\eta}=\frac{1}{\pi} N^{2} a^{2} b c \sum_{p=1}^{\infty} \frac{1}{p^{2}}\left(1+\frac{2 h}{\sqrt{p}} \frac{1+\chi_{p}}{1+\left(1+\chi_{p}\right)^{2}}\right)^{-1} .
$$

At very low concentrations when $\chi_{p} \ll 1$ one has

$$
\frac{\eta(c)-\eta}{\eta}=\frac{1}{\pi} N^{2} a^{2} b c \sum_{p=1}^{\infty} \frac{1}{p^{2}}\left(1+\frac{h}{\sqrt{p}}\right)^{-1} .
$$

Due to the dependence on $h$, the difference between this and the classical result [4] can be notable. So, for a polymer with small $h$, the ratio of the intrinsic viscosity $[\eta]_{h}=\lim _{c \rightarrow 0}[\eta(c)-\eta] /(\eta c)$ at $h<1$ (when the polymer is assumed to be the Rouse one) to that with $h=0$ changes as a function of $h$ from 1 to $\approx 0.55$, at $h=0.5$ being 30 per cent smaller than in the case of a pure Rouse polymer. For a very large $h$, the intrinsic viscosity is $[\eta]_{h \gg 1}=3 \sqrt{2 / \pi} R_{\mathrm{G}}^{3} \zeta(3 / 2)=6.253 R_{\mathrm{G}}^{3}$. 
Considering the viscosity normalized to this expression, one can find that even for rather large $h$ the difference from the traditional result for the pure Zimm polymer is significant. So, at $h=10$, it represents some 25 per cent and it is still about 10 per cent even for $h$ as large as 50 .

One of the important rheological parameters of polymer solutions is the Huggins coefficient $k_{\mathrm{H}}$. It can be determined from the general expression for the viscosity (29), using the intrinsic viscosity $[\eta]_{h}$ at zero concentration (see equation (30)):

$$
\frac{\eta(c)-\eta}{\eta c}=[\eta]_{h}\left(1+k_{\mathrm{H}}[\eta]_{h} c+\cdots\right) \text {. }
$$

We find

$$
k_{\mathrm{H}}=\frac{3 \pi}{2^{3 / 2}} \sum_{p=1}^{\infty} \frac{1}{p^{7 / 2}}\left(1+\frac{h}{\sqrt{p}}\right)^{-2}\left(1+\frac{3}{4 \sqrt{2} h}\right)^{-1}\left[\sum_{p=1}^{\infty} \frac{1}{p^{2}}\left(1+\frac{h}{\sqrt{p}}\right)^{-1}\right]^{-2} .
$$

Thus, for large $h$ (the Zimm case) one has

$$
k_{\mathrm{H}}=\frac{3 \pi}{2^{3 / 2}} \zeta\left(\frac{5}{2}\right) \zeta^{-2}\left(\frac{3}{2}\right) \approx 0.655 .
$$

This value differs from the literature results, see, e.g., [4] where $k_{\mathrm{H}}=0.757$ is given; in [19] one finds $k_{\mathrm{H}}=0.6949$, and in [20] the calculations give the value 0.3787 . The Freed and Edwards theory $[21,22]$ possesses an intrinsic viscosity, which is inconsistent with the Kirkwood-Riseman steady-state limit and gives the hydrodynamic screening even for infinitely dilute solutions (the discussion of this issue has been already given in the paper [5]).

As $h \rightarrow 0$ (the Rouse case), $k_{\mathrm{H}}$ approaches zero as $k_{\mathrm{H}} \approx 2 \pi h \zeta(7 / 2) \zeta^{-2}(2)$ and when $h$ grows, the Huggins coefficient slowly converges to the Zimm limit (33). The difference from this limit is significant in a broad region of $h$, e.g., with the maximum $\approx 1.27$ of the function $k_{\mathrm{H}} / k_{\mathrm{HZimm}}$ at $h=3$, and with $k_{\mathrm{H}} / k_{\mathrm{HZimm}} \approx 1.15$ for $h=20$.

\subsection{Monomer motion}

In connection with the unresolved problem of the dynamic nature of hydrodynamic screening in polymer solutions (see Introduction), it is of special interest to consider the time-dependent quantities describing the polymer behaviour. Among such quantities, the relaxation modulus, which determines the shear stress at shear flows can be easily studied since it is given simply by a sum of exponentials containing the relaxation times from equation (23) [4,8]. Here we shall briefly focus on the simplest (but observable [6]) motion of the end monomer within a polymer coil and calculate its mean square displacement (MSD). The MSD part due to internal modes is $[4,11]$

$$
\left\langle r^{2}(t)\right\rangle_{\mathrm{int}}=\frac{4 N a^{2}}{\pi^{2}} \sum_{p=1}^{\infty} \frac{1}{p^{2}}\left[1-\exp \left(-\frac{t}{\tau_{p}(c)}\right)\right] .
$$

As already shown, with growing concentration $c$, every polymer tends to behave as a Rouse one, which is due to the decrease of the Zimm contribution to the relaxation rates $\tau_{p}^{-1}$. The time dependence of this screening is well displayed considering, e.g., the ratio of the Rouse part of the MSD (i.e., if the polymer was the pure Rouse one, $h=0$ ) to the total MSD in the joint Rouse-Zimm model. This function, $\left\langle r^{2}(t)\right\rangle_{\text {int, } \mathrm{R}} /\left\langle r^{2}(t)\right\rangle_{\text {int }}$, depends on the draining parameter $h$, the concentration $c$, and the time. With the growing $t$, the above relation converges to unity showing the transition to the Rouse behaviour. For example, at a concentration $\widetilde{c}=0.1$ and $h=10$ we have $\left\langle r^{2}(t)\right\rangle_{\text {int, } \mathrm{R}} /\left\langle r^{2}(t)\right\rangle_{\text {int }} \approx 0.75$ at $t=\tau_{1 \mathrm{R}}$, at $t=2 \tau_{1 \mathrm{R}}$ the difference from the Rouse MSD is only about 10 per cent, and at $t=5 \tau_{1 \mathrm{R}}$ the initially Zimm polymer becomes indistinguishable from the Rouse one. When the same relation is considered as a function of $\widetilde{c}$ for different times, one sees that the tendency to approach the Rouse limit with the increase of $\widetilde{c}$ is more and more expressed as the time growths. At long times, as expected, the polymer behaves as the Rouse one already at small concentrations. 


\section{Conclusion}

The behaviour of complex polymer systems that are attractive due to their unusual properties as well as numerous applications cannot be comprehended without understanding the behaviour of a single polymer in a liquid, its interaction with the solvent and with other polymers in dilute solutions. Even in situations when we deal with solutions of flexible and unentangled polymers, a number of open questions do exist and new "puzzles" appear. In our opinion, some of the problems are only due to inappropriate use of the existing theories as well as due to a great influence of simple "universal" laws of polymer behaviour, such as the famous $k^{3}$ law for the first cumulant of the dynamic structure factor or the $t^{\alpha}$ laws for the monomer MSD, where $\alpha=1 / 2$ for the Rouse polymer and 2/3 for the Zimm one. A closer look at these laws shows, however, that their application to real situations is rather restricted and they frequently do not correspond to experimental conditions. The model developed in the present work is not particularly new. This model is partially known (when dealing with the single polymer diffusion) since the publication of the work by Kirkwood and Riseman [23]. As to the internal polymer dynamics, our approach corresponds to that by Dubois-Violette and de Gennes [10] who, however, have assumed that the internal modes of the polymers should behave as the Zimm modes (i.e., with the dispersion $\sim p^{-3 / 2}$, thus neglecting the Rouse contribution, initially being present in their theory. Such a simplification requires quantitative arguments and in many cases it is not substantiated, just like the assumption of the continuous distribution of the internal modes; we believe that this is clearly shown in the present work. To take into account the presence of other coils in the solution, we have used the well-known Debye and Bueche (or Brinkman's) theory for a porous medium. Again, this approach has been already used in the polymer physics. However, according to our earlier results regarding the hydrodynamic theory of the polymer dynamics, we could ultimately build a model that is capable of predicting new results on the fundamental characteristics of polymer behaviour in dilute solutions. Some of the quantities characterizing the polymer solutions (viscosity, Huggins coefficient) could be verified in standard experiments (taking into account the draining parameter). We have also proposed a description of the time dependence tending to hydrodynamic screening in dilute polymer solutions; this effect seems to be suitable for computer simulation studies similar to those in [7].

\section{Acknowledgements}

This work was supported by the Scientific Grant Agency of the Slovak Republic.

\section{References}

1. Balabonov S.M., Ivanova M.A., Klenin S.I., Lomakin A.V., Molotkov V.A., Noskin V.A., Biofizika, 1987, 32, 933.

2. Harnau L., Winkler R., Reineker P., J. Chem. Phys., 1996, 104, 6355.

3. Dünweg B., Reith D., Steinhauser M., Kremer K., J. Chem. Phys., 2002, 117, 914.

4. Doi M., Edwards S.F. The Theory of Polymer Dynamics. Clarendon, Oxford, 1986.

5. Muthukumar M., J. Phys. A: Math. Gen., 1981, 14, 2129.

6. Shusterman R., Alon S., Gavrinyov T., Krichevsky O., Phys. Rev. Lett., 2004, 92, 048303.

7. Ahlrichs P., Everaers R., Dünweg B., Phys. Rev. E., 2001, 64, 040501.

8. Larson R.G., J. Rheol., 2005, 49, 1.

9. Lisy V., Tothova J., Zatovsky A.V., J. Chem.Phys., 2004, 121, 10699.

10. Dubois-Violette E., de Gennes P.-G., Physics, 1967, 3, 181.

11. Tothova J., Brutovsky B., Lisy V., Czech. J. Phys., 2005, 55, 221.

12. Tothova J., Lisy V., Zatovsky A.V., J. Chem. Phys., 2003, 119, 13135.

13. Brinkman H.C, Proc. Amsterdam Acad., 1947, 50, 6.

14. Debye P., Bueche A.M., J. Chem. Phys., 1948, 16, 573.

15. Zatovsky A.V., Lisy V., J. Mol. Liq., 2003, 105, 289.

16. Grosberg A.Yu., Khokhlov A.R. Statistical Physics of Macromolecules. Nauka, Moscow, 1989 (in Russian). 
17. Zatovsky A.V., Levin M.V., Lisy V., Physics of Aerodisperse Systems, 2001, 38, 228 (in Russian).

18. Zhao H. Beckham W., Ricks H.L., Bunz U.H.F., Polymer, 2005, 46, 4839.

19. Muthukumar M., J. Chem. Phys., 1983, 79, 4048.

20. Muthukumar M., Freed K.F., Macromolecules, 1978, 11, 843.

21. Freed K.F., Edwards S.F, J. Chem. Phys., 1974, 61, 3626.

22. Freed K.F., Edwards S.F, J. Chem. Phys., 1975, 62, 4032.

23. Kirwood J.G., Riseman J., J. Chem Phys., 1948, 16, 565.

\title{
Об’єднана теорія Роуза-Зіма динаміки полімерів у розчинах
}

\author{
В.Ліси ${ }^{1}$, Й.Тотова ${ }^{1}$, О.Затовський ${ }^{2}$ \\ 1 Фізичний факультет, Університет ім. П.Й.Шафаріка, \\ 04054 Кошіце, Словацька Республіка \\ 2 Одеський національний університет ім.І.І.Мечникова, \\ факультет теоретичної фізики \\ 65026 Одеса, вул. Дворянська, 2, Україна
}

Отримано 11 липня 2005 р.

\begin{abstract}
Ми пропонуємо теорію динаміки полімерів у слабких розчинах, у якій популярні моделі Зіма та Роуза $€$ просто граничнии випадками з безмежно великим та малим параметром просочування. Рівняння руху для сегментів полімера розв'язуються разом з рівнянням Брінкмана для швидкості розчинника, що враховує присутність інших полімерних спіралей в розчині. Отримано рівняння для нормальних мод полімера та знайдено відповідні часові кореляційні функції. Тенденція до залежного від часу гідродинамічного екранування демонструється на дифузії полімерів, а також на релаксації їх внутрішніх мод. При зростаючій концентрації спіралей у розчині вони обидва показують перехід до точно поведінки Роуза. Розраховано зсувну в'язкість розчину, коефіцієнт Хаггінза та інші величини та показано, що вони помітно відрізняються від відомих результатів.
\end{abstract}

Ключові слова: полімери, динаміка, слабкі розчини, реологія

PACS: $36.20 . F z, 36.20 .-r$, 82.35.Lr, 82.37.-j, 83.80.Rs 